\title{
On Evolution Properties of E-bound Growing Network Models
}

\author{
Xia Liu ${ }^{1}$, Bing Yao ${ }^{1, \mathrm{a}}$, Xiaomin Wang ${ }^{1}$, Xiyang Zhao ${ }^{1}$, Mingjun \\ Zhang $^{2}$ \\ ${ }^{1}$ College of Mathematics and Statistics, Northwest Normal University, \\ Lanzhou, Gansu 730070, CHINA \\ ${ }^{2}$ School of Information Engineering, Lanzhou University of Finance and \\ Economics, CHINA \\ ${ }^{a}$ Correspond author's email: yybb918@163.com \\ bemail: yybm918@163.com
}

\begin{abstract}
.
A class of bound growing network models has been defined in this article. We determine some properties of the new models, such as scale-free, power law distribution, degree cumulative distributionn and clustring coefficient. Furthermore, we design two stochastic methods for the new models.

Keywords: growing network; edge-cumulative distribution; scale-free networks.
\end{abstract}

\section{Introduction}

One have known that most real-world networks are not random network, but scale-free that a few nodes tend to have a lot of connections and most of the nodes rarely. In other words, scale-free networks has severe heterogeneity resistance, the connection status (degrees) between the nodes which have seriously non-uniform distribution: nodes in the network with a small number of points called hub points have many connections and most of the nodes connected to only a small amount. Minority hub point plays a leading role on scale-free network. Robustness and fragility of the Internet as a special large-scale basic features, reflect the significant differences between the random graph networks and scale-free network topology characteristics. Because of the presence of the power-law distribution of scale-free networks greatly raising the possibility of nodes exist high connections. Therefore, scale-free networks show the robustness against deliberate attacks and fragility against random failures [1]. It has a very big impact on fault tolerance and anti-attack capability. Study shows that scale-free networks have highly fault-tolerant, but weak resistance based on the selective attack node having big degree. 
In recent decade years, there are many literature for understanding scale-free networks in terms of graph theory. So, one have built up graph models of growing type and show the relevant parameters of the graphs models (i. e. diameter, average distance, clustering, degree distribution and degree correlation, and so on) (Ref. [2]-[9]). Barabasi and Albert [1] show a type of growing networks; Comellas et al. [2,3] show a class of growing network models by graph theory; Zhang et al.[12] show the scale-free of scale-free networks. Building up network models is an important way to visualize some networks and to understand or approximate real networks. Clearly, network models having good behaviors may used to construct real networks in the world. The purpose of this paper is to explore the nodes of network models have what behaviors such that as hub nodes and blocky impact in the models. Thus, we first construct a growing network model, then carry out the basic parameters and verify its scale-free (Ref. [5]-[11]). We use notation $|X|$ to denote the number of elements of a set $X$ in this article.

\section{E-bound growing network models}

We construct our E-bound growing network models $N(t)$. Based on the initial network model $N(0), N(t)$ is obtained from $N(t-1)$ by only doing the adding-operation, and called $N(t)$ an E-bound growing network model (E-BGN-model for short). Let $X_{t}$ be the set of nodes newly added into $N(t-1)$, so that $V(t)=V(\mathrm{t}-1) \cup X_{t}$; and let $Y_{t}$ be the set of edges newly added into $N(t-1)$, thus $E(t)=E(t-1) \cup Y_{t}$. The notation $B(t)$ denotes the set of bound-edges of $N(t)$. It is not hard to obtain $|B(t)|=2 r|B(t-1)|$ for $t \geq 1$. Write the node-number $n_{v, s}=|V(s)|$ and the edge-number $n_{e, s}=|E(s)|$ at time step $s \geq 0$. By

$$
n_{v, 1}=n_{v, 0}+m n_{e, 0}, n_{e, 1}=n_{e, 0}+2 m n_{e, 0}=(2 m+1) n_{e, 0},\left|Y_{t}\right|=2\left|X_{t}\right|
$$

and $|B(1)|=2 r m n_{e, 0}$, we can compute the node-number $n_{v, t}$ and edge-number $n_{e \text {, }}$ $t$ as follows.

$$
\begin{aligned}
& n_{v, \mathrm{t}}=n_{v, t-1}+m n_{e, 0}(2 \mathrm{r})^{t-1}=n_{v, 0}+m n_{e, o} \frac{(2 \mathrm{r})^{t}-1}{2 r-1}, \\
& n_{\mathrm{e}, \mathrm{t}}=n_{\mathrm{e}, t-1}+2 m n_{e, 0}(2 \mathrm{r})^{t-1}=n_{e, o}\left[1+2 m \frac{(2 \mathrm{r})^{t}-1}{2 r-1}\right] .
\end{aligned}
$$

Every E-BGN-model $N(k)$ has $|B(k)|=n_{e, 0}(2 r)^{k}$ bound-edges and the number of nodes newly added that is

$$
\left|X_{t}\right|=n_{v, k}-n_{v, k-1}=m n_{e, 0}(2 r)^{k-1}, k=1,2, \ldots, t .
$$

1. Degree spectrum. Let $d_{1}, d_{2}, \ldots, d_{a}$ be the different degrees of nodes of the initial network $N(0)$, without loss of generality, $d_{1}>d_{2}>\ldots>d_{a}$. Let $n_{k}(d)$ be the

number of nodes of degree $d$ in $N(k)$. Hence, $2 n_{e, 0}=\sum_{j=1}^{a} d \cdot n_{0}\left(d_{j}\right)$ by the basic theorem of graph theory. 
(1) A node $u$ of degree $d_{j}$ in $N(0)$ has degree $d_{j}+m d_{j}$ in $N(1)$, and it distributes $r d_{j}$ bound-edges to $N(1)$. In this way, the node $u$ of $N(k)$ with $k \geq 1$ has degree $d_{j}\left(1+m \sum_{i=0}^{k-1} r^{i}\right)$ and it distributes $r^{k} d_{j}$ bound-edges to $N(k)$. Let $I\left(d_{j}, x\right)=d_{j}\left(1+m \sum_{i=0}^{k-1} r^{i}\right)$ for $x=1,2, \ldots, a$. We present a table:

\begin{tabular}{|c|c|c|c|c|c|}
\hline$d$ & $I\left(d_{a}, t\right)$ & $I\left(d_{a-1}, t\right)$ & $\ldots$ & $I\left(d_{2}, t\right)$ & $I\left(d_{1}, t\right)$ \\
\hline$n_{t}(d)$ & $N_{0}\left(d_{a}\right)$ & $N_{0}\left(d_{a-1}\right)$ & $\ldots$ & $N_{0}\left(d_{2}\right)$ & $N_{0}\left(d_{1}\right)$ \\
\hline
\end{tabular}

(2) A node $w_{j}^{k}$ added newly into $N(k)$ at time step $k$ has degree 2. If it is selected as the common end of two bound-edges $u^{k} w_{j}^{k}$ and $w_{j}^{k} v^{k}$ into $N(k+1)$, then $w_{j}^{k}$ has degree $2+2 m$ and distributes $2 r$ bound-edges to $N(k+1)$. Thereby, in $N(t)$ at time step $t$, the node $w_{j}^{k}$ has its own degree $2+2 m \sum_{i=0}^{t-k-1} r^{i}$ and distributes $2 r^{t-k}$ bound-edges to $N(t)$.

(3) In general, the number of nodes of degree 2 in $N(t)$ is equal to $A^{*}=\left|X_{t}\right|+\frac{m-r}{m} \sum_{j=1}^{t-1}\left|X_{j}\right|$. For the purpose of simplicity we define two functions $f(x)=2+2 m \sum_{i=0}^{x} r^{i}$ and $F(x)=r f(x) / m$, where $\left|X_{k}\right|$ is defined in Eq. (3). By the above facts, we are not difficult to obtain the degree spectrum of an E-BGN-model $N(t)$ at time step $t \geq 1$ as follows:

\begin{tabular}{|c|c|c|c|c|c|c|c|c|}
\hline$d$ & 2 & $f(0)$ & $f(1)$ & $\ldots$ & $f(t-2)$ & $I\left(d_{a}, t\right)$ & $\ldots$ & $I\left(d_{a, 1}\right)$ \\
\hline$n_{t}(d)$ & $A^{*}$ & $F(t-1)$ & $F(t-2)$ & $\cdots$ & $F(1)$ & $N_{0}\left(d_{a}\right)$ & $\ldots$ & $N_{0}\left(d_{1}\right)$ \\
\hline
\end{tabular}

Clearly, an E-BGN-model $N(t)$ at time step $t \geq 1$ has its own maximum degree $d_{1}\left(1+m \sum_{i=0}^{t-1} r^{i}\right)$ and minimum degree 2. By the degree spectrum, we can compute:

1) The average degree $\langle k\rangle$ of $N(t)$ holds $\langle k\rangle \rightarrow 4$ as $t \rightarrow \infty$, which shows that $N(t)$ is a sparse network model with as few links as possible.

2) We have $2\left|Y_{t}\right| /\left|X_{t}\right|=4$, which shows that $2\left|Y_{t}\right| /\left|X_{t}\right|$ is equivalent to $\langle k\rangle$ as $t$ tends to infinity. In other words, the density $\langle k\rangle$ of $N(t)$ is equivalent to the density $2\left|Y_{t}\right| /\left|X_{t}\right|$ of growing procedure.

2. Distributions. As known, the topological structures of network models can be described by the various distributions. 
Degree distribution. Degree distribution is one of most important topological characters of a network.The degree of node $i$ is defined as the number of direct connections it has to other nodes. We, very often, use $P(k)$ to be the probability of nodes having degree $k$ in an E-BGN-model $N(t)$ at time step $t \geq 1$. Note that the degree spectrum of $N(t)$ is discrete. By the method used in [9] we compute $P(k)$ in the following. Let $k=2+2 m \sum_{j=1}^{t-\tau} r^{t-\tau-j}$, where $\tau$ is the time step that is the node entering into $N(\tau)$. So, we obtain a function $h(x)=[\ln (k r-k-x r+2 m+2)-\ln 2 m] / \ln r$ for $r \geq 2$. According to Eq.(3), we get

$$
P\left(k^{*}>k\right)=\frac{1}{n_{v, t}}\left(n_{v, 0}+\frac{r}{m} \sum_{k=1}^{\tau}\left|X_{k}\right|\right) \propto \frac{r}{m}(2 r)^{\tau-t}
$$

Plugging $t-\tau=h(2)$ into the above equation enables us to obtain $(2 r)^{\tau-t} \propto(2 r)^{-h(2)}$. Furthermore,

$$
P(k)=P\left(k^{*}>k-1\right)-P\left(k^{*}>k\right) \propto \frac{r}{m}\left[(2 r)^{-h(3)}-(2 r)^{-h(2)}\right]
$$

Thereby, the E-BGN-model $N(t)$ obeys the exponential distribution, so it is scale-free.

Clustering distribution. For each node $I$ of an E-BGN-model $N(t)$, we use $E_{i}$ to denote the number of edges between $k_{i}$ nodes that are adjacent to the node $i$. Clearly, $k_{i}\left(k_{i}-1\right) / 2$ is defined as the maximum number of edges between $k_{i}$ nodes. Hence, the clustering coefficient $C_{i}$ of the node $i$ is defined as $C_{i}=2 E_{i} / k_{i}\left(k_{i}-1\right)$ which means the node $i$ measures how many pairs of its neighbors are directly connected.The clustering coefficient $\langle c\rangle$ of the model $N(t)$ is the average of the local clustering coefficient over all node in the network, it defined as $\langle c\rangle=\left(\sum_{i \in V(G)} C_{i}\right) / n_{v, t}$. At time step $t$, the clustering coefficient $\langle c\rangle$ of an E-BGN-model $N(t)$ can be computed for $r \geq 2$ as follows. 


$$
\langle c\rangle=\frac{1}{n_{v, t}} \sum_{i \in V(t)} C_{i}=\frac{1}{n_{v, t}}\left[\sum_{1 \leq \tau \leq t} \frac{2 r\left|X_{\tau}\right|}{m k_{\tau}}+\sum_{i=0}^{n_{v}, 0} \frac{2}{I\left(d_{i}, t\right)}\right] \propto 1-\frac{1}{4 m r}
$$

Thus, we can see $0.5 \leq\langle c\rangle \leq 0.6$ as $t \rightarrow \infty$, which means that the E-BGN-model $N(t)$ has higher clustering distribution for big $m$.
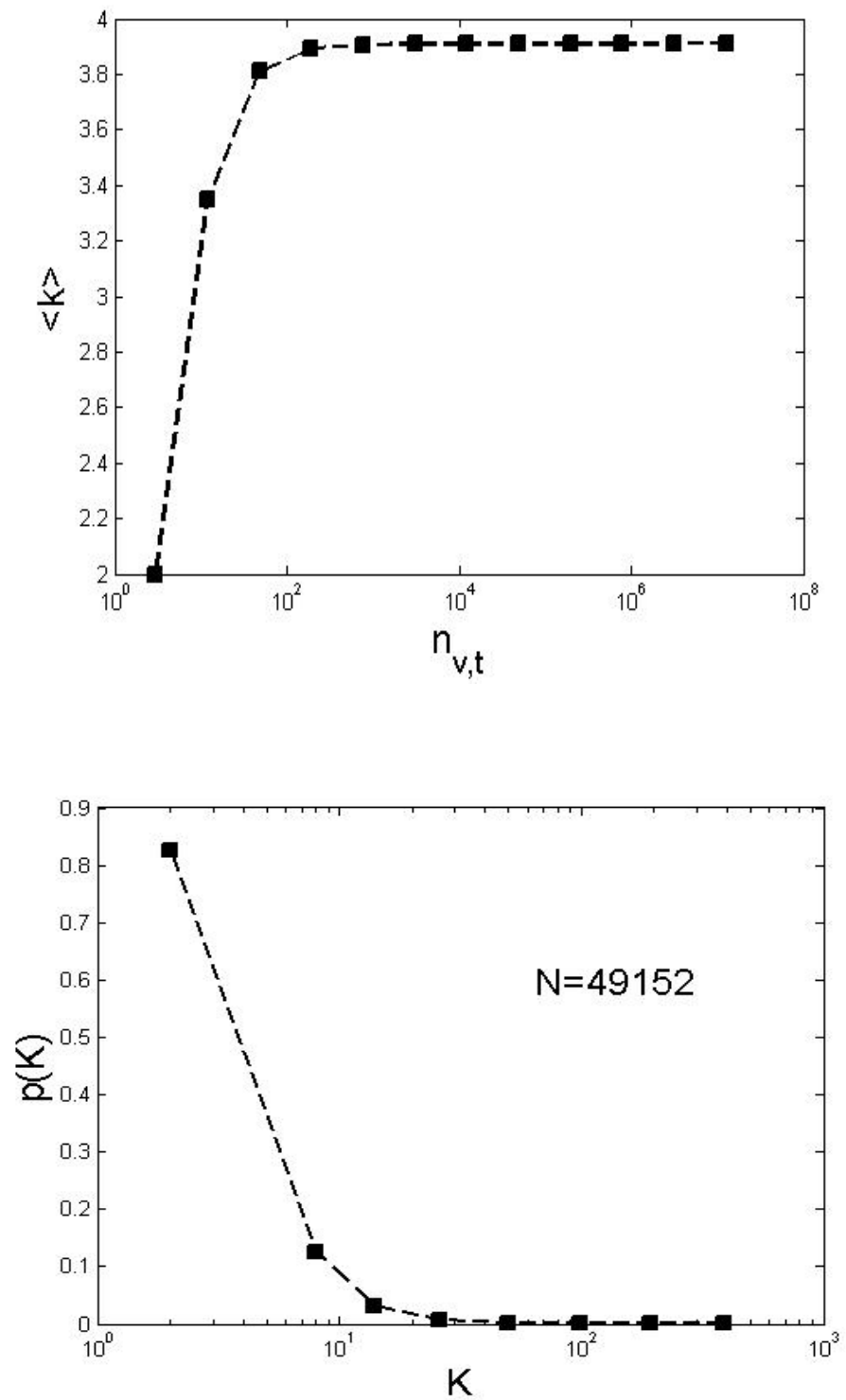


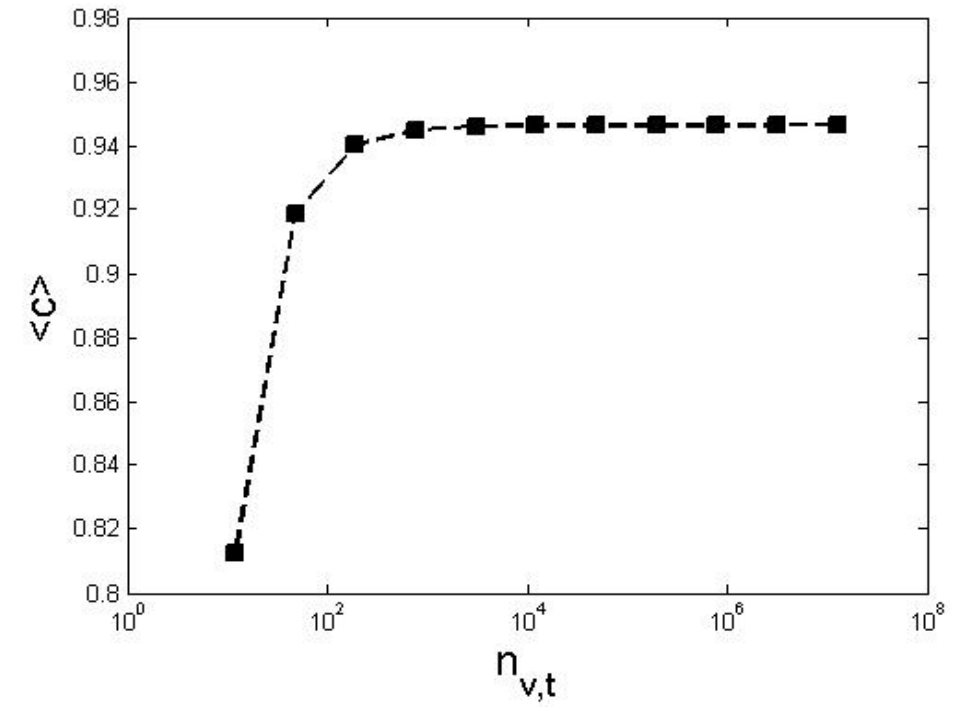

Figure-1 The left describes the tendency of the average degree $\langle k\rangle$; the middle is for degree distribution; the right describes the tendency of the clustering coefficient $\langle c\rangle$.

Edge-cumulative distribution. We are motivated from the degree cumulative distribution, and define a new cumulative distribution. As $0<\tau<t$, we define a new stochastic technique $P_{e-\text { cum }}(k)$ of an E-BGN-model $N(t)$ and called it the edge-cumulative distribution:

$$
\begin{gathered}
P_{e-\text { cum }}(k)=\frac{1}{n_{e, t}} \sum_{r=0}^{\tau} n_{e, r} \propto \frac{2}{2 r-1}(2 r)^{\tau-t} \\
\tau=t-\left[\frac{2 r}{\ln (2 r)}+\frac{1}{\ln (2 r+m)}\right] \ln k, \text { we have } \\
P_{e-\text { cum }}(k)=\frac{1}{n_{e, t}} \sum_{r=0}^{\tau} n_{e, r} \propto \frac{2}{2 r-1} k^{-2 r-\frac{\ln (2 r)}{\ln (2 r+m)}}
\end{gathered}
$$

Applying

It shows that $P_{e-\text { cum }}(k)$ obeys the power law degree distribution. For integers $m \geq 1$, the case $r=1$ implies $2<\gamma_{k}<3$, where $\gamma_{k}=2+\frac{\ln 2}{\ln (2 r+m)}$, and $P_{e-\text { cum }}(k)$ is influenced only by the parameter $r$. 


\section{Summary}

We construct the E-BGN-model $N(t)$ and show several distributions for our models. As furtherwork, $N(t)$ can be considered as $\left(\alpha_{k}, \beta_{k}\right)$-model. The total number $S_{N}(\leq k)$ of nodes of degrees no more than $k$ in $N(t)$ is defined as $S_{N}(\leq k)=\sum_{d \leq k} n_{d}(t)$ and the sum of degrees of these nodes is defined as $D_{N}(\leq k)=\sum_{d \leq k} d \cdot n_{d}(t)$. Let $S_{N}(>k)=n_{v}, t-S_{N}(\leq k)$ and $D_{N}(>k)=n_{e, t}-D_{N}(\leq k)$. Notice that $k=2+2 m \sum_{j=1}^{t-\tau} r^{t-\tau-j}$, according to the construction of $N(t)$. We can calculate $S_{N}(\leq k) / n_{v, t} \propto 1-P\left(k^{*}>k\right) / 2=1-\alpha_{k}$, and $S_{N}(\leq k) / n_{v}, t \propto r(2 r)^{-h(2)-2} / m=\alpha_{k}$. We can compute $D_{N}(\leq k) / n_{e}, t \infty 1-(2 r)^{-h(2)} /(r-1)(2 r-1)$. Thereby, we call $N(t)$ an $\left(\alpha_{k}, \beta_{k}\right)$-growing network model. Clearly, and claim that $\alpha_{k}, \beta_{k}$ obeys the power law distribution. Another working direction should be mentioned is that we have done rewiring edges or removing/adding edges to out models in order to produce randomized network models, and have found the maximum leaves spanning trees in our models introduced here.

Acknowledgment. This work was funded partly by the National Natural Science Foundation of China under Grant No. 61163054, No. 61363060 and No. 61163037.

\section{Reference}

[1] Albert-aszlo Barabasi and Reka Albert. Emergence of scaling in random networks. Science Vol 286(1999)509-512.

[2] Francesc Comellas, Guillaume Fertin, Andre Raspaud, Recursive graphs with small-world scale-free properties, Physical Review E 69, 03710-1, 037104-4 4 (2004)

[3] Francesc Comellas, Alicia Miralles, Vertex labeling and routing in self-similar outerplanar unclustered graphs modeling complex networks, Journal of Physics A: Mathematical and Theoretical, 2009, 42, pp: 425001.

[4] L. da F. Costa F. A. Rodrigues G. Travieso P. R. Villas Boas, Characterization of Complex Networks: A Survey of measurements, arXiv:cond-mat/0505185v5 Aug 2006. 
[5] Xia Liu, Bing Yao, Wanjia Zhang, Xiang-en Chen, Xiaomin Zhang. Topological Structure and Spanning Trees of Rectangular growing network models. Applied Mechanics and Materials Vols. 644-650 (2014) pp 1805-1808. DOI:10.4028/www.scientific.net/AMM.644-650.1805.

[6] Xia Liu, Bing Yao, Wanjia Zhang, Xiang'en Chen, Xinsheng Liu, Ming Yao. Uniformly Bound-Growing Network Models And Their Spanning Trees.The 2014 International Conference on Information and Communications Technologies (ICT2014) will be held 16th-18th May 2014 in Nanjing, China. 2014, pp35-38

[7] Bing Yao, Hongyu Wang, Ming Yao, Xiang'en Chen, Chao Yang and Xiaomin Zhang. On The Collapse Of Graphs Related to Scale-Free Networks. Proceeding of ICIST2013.Third International Conference on Information Science and Technology March 23-25, 2013;738-743. 978-1-4673-2764-0/13 IEEE

[8] Bing Yao, Ming Yao, Xiang-en Chen, Xia Liu, Wan-jia Zhang. Research on Edge-Growing Models Related with Scale-Free Small-World Networks. Applied Mechanics and Materials, Volumes 513-517, pp 2444-2448. DOI:10.4028/www.scientific.net/AMM.513-517.2444

[9] Bing Yao, Chao Yang, Ming Yao, Hongyu Wang, Xiang'en Chen, Xiaomin Zhang, Mogang Li. Graphs As Models of Scale-free Networks. Applied Mechanics and Materials, Vol.380-384(2013) pp2034-2037. DOI: 10.4028/www.scientific.net/AMM.380-384.2034

[10] Bing Yao, Ming Yao, Sihua Yang, Xiang'en Chen, Xiaomin Zhang. Labelling Edges of Models from Complex Networks. Applied Mechanics and Materials, Volumes 513-517, pp1858-1862. DOI:10.4028/www.scientific.net/AMM.513-517.1858

[11] Chao Yang, Bing Yao, Hongyu Wang, Xiang'en Chen, Sihua Yang. Graph Colorings Applied In Scale-Free Networks. Advanced Materials Research Volumes 760-762 (2013),pp2199-2204.DOI: 10.4028/www.scientific.net/AMR.760-762.2199

[12] Zhang Zhongzhi, Rong Lili, Guo Chonghui. A deterministic small-world network created by edge iterations, Physica A, 2006, 363(2):567-572. 